\title{
SPACE CHARGE LIMITED CURRENTS IN ANTHRACENE CRYSTALS UNDER MODULATED ILLUMINATION
}

\author{
A. UZARCZYK AND J. GodlewSKI \\ Department of Molecular Physics, Technical University of Gdanisk \\ Narutowicza 11/12, 80-952 Gdanisk, Poland
}

Space charge limited currents have been examined in anthracene crystals illuminated with a chopped beam of monochromatic light $(\lambda=420 \mathrm{~nm})$. The beam was uniformly absorbed in the anthracene crystal. The primary objective of our research was to determine mechanisms responsible for the release and trapping of charge carriers in the case of space charge limited currents as a function of time. The experimental results concern the dependences of photocurrents on the frequency of light chopping, light intensity, voltage and current decay after illumination. Photocurrent decay as a function of time was exponential for short time periods (i.e. for milliseconds), while for longer periods it was described by the power function $t^{-1 / l}$, where parameter $l$ is the characteristic parameter of the exponential distribution of traps.

PACS numbers: $72.40 .+w$

\section{Introduction}

Space charge limited currents (SCLC) in insulators are responsive to illumination, which causes charge carrier detrapping by interaction with photons or excitons. In molecular materials due to exciton generation under illumination, the trapped carrier-exciton interaction, is of special importance. Charge carrier detrapping by excitons shortens the lifetime of trapped charge carriers in traps and thus increases the space charge limited current. Photocurrent so obtained is called photo-enhanced current and was analysed in several papers [1-4].

The objective of this paper is to analyse time dependence of transition currents from the photo-enhanced currents to dark currents in space charge limited conditions. It has been experimentally accomplished by modulation of illumination and screening the light beam reaching the anthracene sample with the hole injecting (CuI) electrodes attached. 


\section{Theoretical basis}

Theoretical and experimental analysis of transition currents will be limited to the case of exponential energy distributions of traps of the following form $[1,5]$ :

$$
h(E)=\frac{E}{l k T} \exp \left(-\frac{H}{l k T}\right),
$$

where $H$ is the total concentration of traps and $l-$ the characteristic parameter of the trap distribution.

In the context of the exponential trap distribution, thermally stimulated SCLC has the following form:

$$
j=\frac{N_{\mathrm{eff}} e \mu}{H^{l}}\left(\frac{\varepsilon \varepsilon_{0}}{e}\right)^{l}\left[\frac{l^{2} \sin (\pi / l)}{(l+1) \pi}\right]^{l}\left(\frac{2 l+1}{l+1}\right)^{l+1} \frac{U^{l+1}}{d^{2 l+1}},
$$

where $N_{\text {eff }}$ is the effective density of states, $\mu$ - the mobility of charge carriers, $\varepsilon$ - the dielectric constant, $e$ - electron charge, $U$ - voltage applied to the sample, and $d-$ thickness of the sample.

In the presence of illumination at low absorption of the light, the photo-enhanced current is expressed as follows $[2,3]$ :

$$
j_{\mathrm{ph}}=\frac{9}{8} \varepsilon \varepsilon_{0} \mu \frac{N_{\mathrm{eff}} l \sin (\pi / l)}{H \pi}\left(\frac{A \kappa I_{0}}{\nu}\right)^{1-1 / l} \frac{U^{2}}{d^{3}},
$$

where $A$ is a certain constant connected with the charge carrier detrapping by excitons, $\kappa-$ the light absorption coefficient, $I_{0}$ - light intensity of the beam, and $\nu$ - the collision factor.

The photo-enhanced current (3) is always larger than the thermally stimulated current (2). The decay of SCLC transition current should be expected to depend on:

(i) lifetimes of singlet $\left(\tau_{\mathrm{S}}\right)$ and triplet $\left(\tau_{\mathrm{T}}\right)$ excitons $[2,4,8]$,

(ii) the mechanism of space charge transport - with or without dispersion in the sample $[6,7]$,

(iii) the rate of thermal detrapping of charge carriers trapped under illumination.

The nature of photocurrent decay will be analysed on the basis of experimental results obtained for anthracene crystals under SCLC conditions.

\section{Experimental results}

Examples of experimental SCLC dependences for an anthracene crystal, dark current and current under stationary illumination, are shown in Fig. 1. The value of the parameter $l=4.3$ was found from the dark characteristic, in accordance with the dependences following from Eqs. (1) and (2).

If the light is modulated, the decay of transition current within the range of miliseconds is as it shown in Fig. 2. It follows from Fig. 2 that the transition current decays exponentially with time periods comparable with the lifetime of triplet excitons in anthracene $\left(\tau_{\mathbf{T}} \approx 20 \mathrm{~ms}\right.$ [8]), while there is no evidence of the influence of singlet excitons on charge carrier detrapping. 


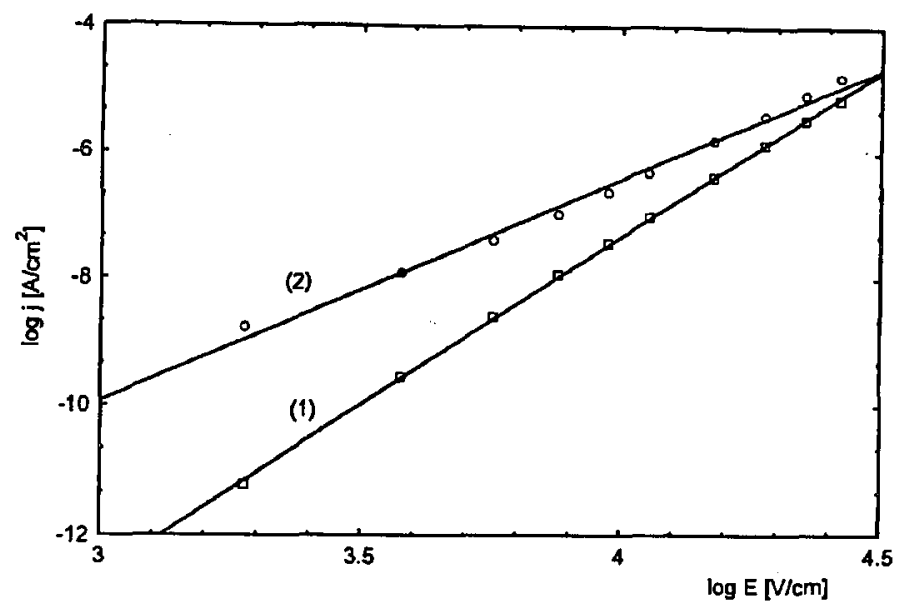

Fig. 1. Density of dark current (1) and current under illumination (2) with wavelength $\lambda=420 \mathrm{~nm}$, as a function of electric field in an anthracene crystal: the thickness of the sample $d=60 \mu \mathrm{m}$.

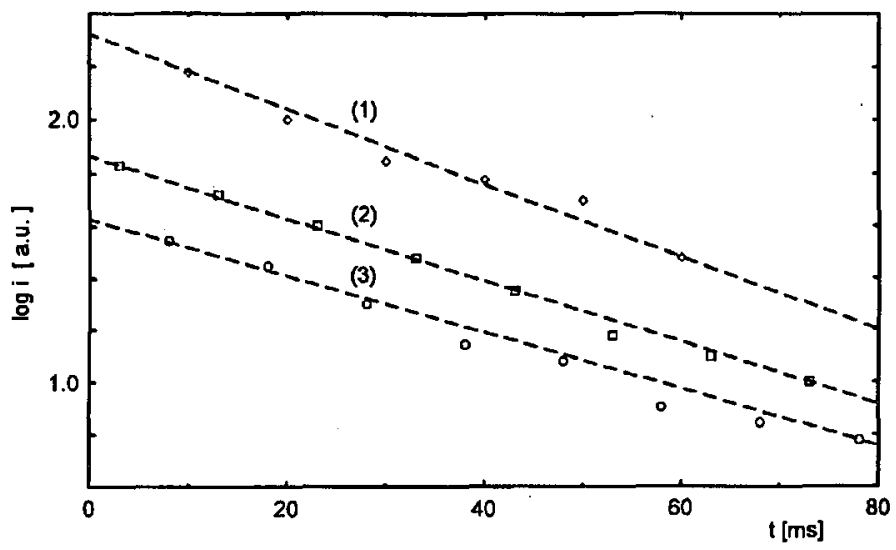

Fig. 2. Transition current decay in anthracene crystals as a function of time (for a miliseconds range) for various electric fields: $E=10^{4} \mathrm{~V} / \mathrm{cm}(1), E=7 \times 10^{3} \mathrm{~V} / \mathrm{cm}(2)$, $E=6 \times 10^{3} \mathrm{~V} / \mathrm{cm}(3)$, the thickness of the crystal $d=60 \mu \mathrm{m}$, the wavelength of exciting light $\lambda=420 \mathrm{~nm}$.

After screening the light, the decay of transition current lasts for seconds, followed by the photocurrent's virtual return to the dark current. Current decays of the longer time periods are shown in Fig. 3. The broken line represents the time-current dependence in the following form [9]:

$$
j(t) \simeq \frac{n_{0} e d H \nu^{1-1 / l}}{N_{\mathrm{eff}}} t^{-1 / l} \propto t^{-1 / l},
$$

where $n_{0}$ is the concentration of the free charge carriers at $t=0$. 


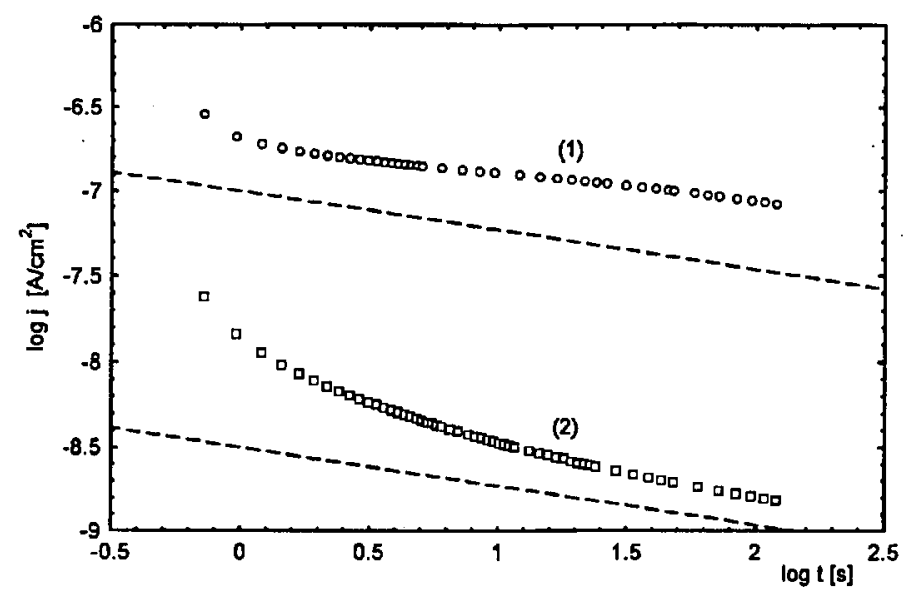

Fig. 3. Transition current decay in anthracene crystals as a function of time (for a seconds range) for various electric fields: $E=7 \times 10^{3} \mathrm{~V} / \mathrm{cm} \mathrm{(1),} E=6 \times 10^{3} \mathrm{~V} / \mathrm{cm}(2)$, the thickness of the crystal $d=60 \mu \mathrm{m}$, the wavelength of exciting light $\lambda=420 \mathrm{~nm}$. The broken line represents the time-dependent current according to Eq. (4) (see text).

The above dependence has been obtained on the assumption that transition current is due to thermal detrapping of charge carriers trapped during the flow of stationary photo-enhanced current.

However, it is not the time-current dependence following from the mechanisms of charge carrier transport found for transition currents in the papers $[6,7]$.

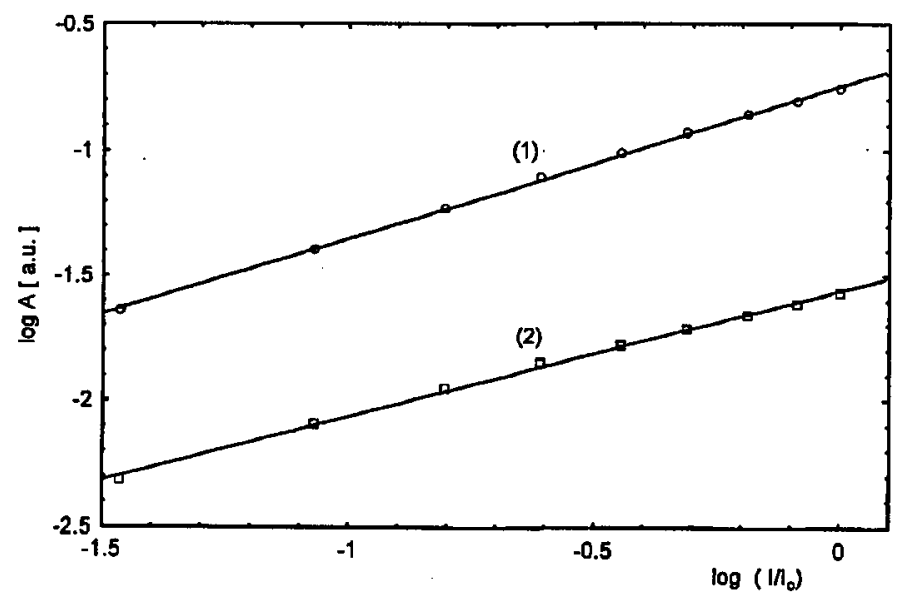

Fig. 4. Dependence of the amplitude of transition current as a function of relative light intensity for electric field $E=6 \times 10^{3} \mathrm{~V} / \mathrm{cm}$, with various modulation frequencies of the light beam: $f=6.8 \mathrm{~Hz}(1)$ and $f=116 \mathrm{~Hz}(2) ; \lambda=420 \mathrm{~nm}, d=60 \mu \mathrm{m}$. 


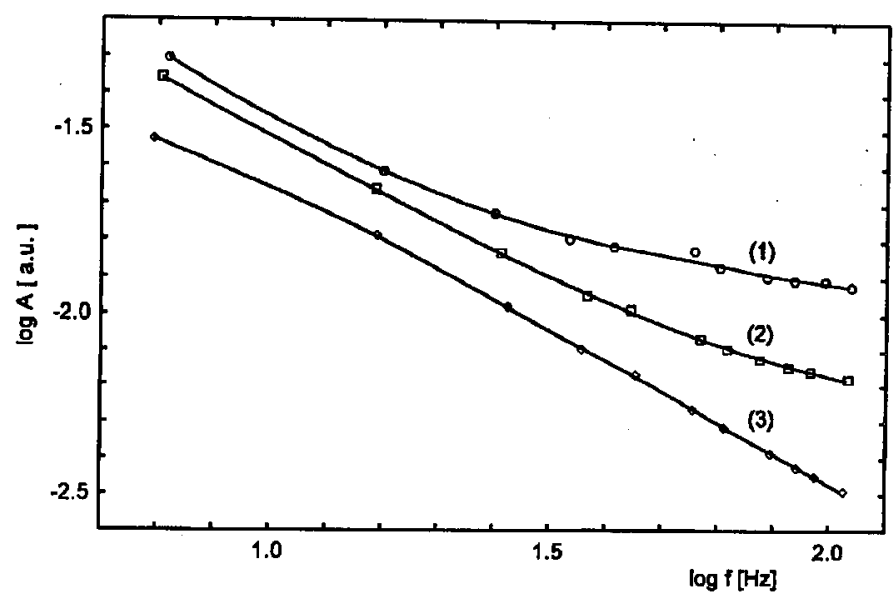

Fig. 5. Dependence of the amplitude of transition current as a function of frequency with various intensities of electric field: $E=10^{4} \mathrm{~V} / \mathrm{cm}(1), E=7 \times 10^{3} \mathrm{~V} / \mathrm{cm}$ (2), $E=6 \times 10^{3} \mathrm{~V} / \mathrm{cm}(3) ; \lambda=420 \mathrm{~nm}, d=60 \mu \mathrm{m}$.

Dependences of transition currents as functions of illumination intensity and modulation frequency of the light beam have also been examined and are shown in Figs. 4 and 5 . It follows from these dependences that the amplitude of the transition current is sublinearly dependent on light intensity and decreases - linearly at most - with the beam's frequency. The sublinear dependence of the amplitude of transition current on light intensity is in accordance with the sublinear dependence of the stationary photo-enhanced current on the light intensity (3), while the linear dependence of the amplitude of the modulated current is explicable under the assumption that an anthracene crystal acts as a light detector with a long time constant (of the order of seconds).

\section{Conclusions}

The analysis of the modulated photo-enhanced currents in anthracene crystals under SCLC condition suggests that time dependences of these photocurrents are due to the long-lasting processes connected with the lifetime of triplet excitons and the mechanisms of charge carrier detrapping.

The exponential dependences of transition currents as a function of time in the range of miliseconds result from the exponential decay of triplet exciton concentrations, while the dependence (4) for long time periods is due to detrapping of charge carriers from traps out of balance of the trapping-detrapping kinetics.

\section{Acknowledgment}

This work was supported in part by the State Committee for Scientific Research (Republic of Poland) project nr 204599101. 


\section{References}

[1] P. Mark, W. Helfrich, J. Appl. Phys. 33, 205 (1962).

[2] H. Bauser, H.H. Ruf, Phys. Status Solidi 32, 135 (1969).

[3] J. Godlewski, J. Kalinowski, Phys. Status Solidi A 53, 161 (1979).

[4] J. Godlewski, J. Kalinowski, P.Di Marco, Mol. Cryst. Liq. Cryst. 228, 61 (1993).

[5] J. Godlewski, J. Kalinowski, Solid State Commun. 25, 473 (1978).

[6] H. Scher, E.W. Montroll, Phys. Rev. B 12, 2455 (1975).

[7] T. Tiedje, A. Rose, Solid State Commun. 37, 49 (1980).

[8] M. Pope, R. Selsby, Chem. Phys. Lett. 14, 226 (1972).

[9] A. Uzarczyk, J. Godlewski, to be published. 